\title{
Micro-spherical cochleate composites: method development for monodispersed cochleate system
}

Kalpa Nagarsekar ${ }^{1^{*}}$, Mukul Ashtikar ${ }^{1^{*}}$, Frank Steiniger ${ }^{2}$, Jana Thamm ${ }^{1}$, Felix H. Schacher ${ }^{3,4}$, Alfred Fahr ${ }^{1 * *}$

${ }^{1}$ Lehrstuhl für Pharmazeutische Technologie, Institut für Pharmazie, Friedrich-Schiller-Universität Jena, Lessingstraße 8, 07743 Jena, Germany

${ }^{2}$ Elektronenmikroskopisches Zentrum, Universitätsklinikum Jena, Ziegelmühlenweg 1, 07743 Jena, Germany

${ }^{3}$ Institut für Organische Chemie und Makromolekulare Chemie, Friedrich-Schiller-Universität Jena, Lessingstraße 8, 07743 Jena, Germany

4 Jena Center for Soft Matter, Friedrich-Schiller-Universität Jena, Philosophenweg 7, 07743 Jena, Germany

*These authors have contributed equally in this study and in the preparation of this manuscript.

**Corresponding Author:

Prof Dr Alfred Fahr,

Lehrstuhl für Pharmazeutische Technologie,

Institut für Pharmazie,

Friedrich-Schiller-Universität Jena,

Lessingstraße 8,

07743 Jena, Germany

Phone: +49 3641949901

Email: Alfred.Fahr@uni-jena.de 


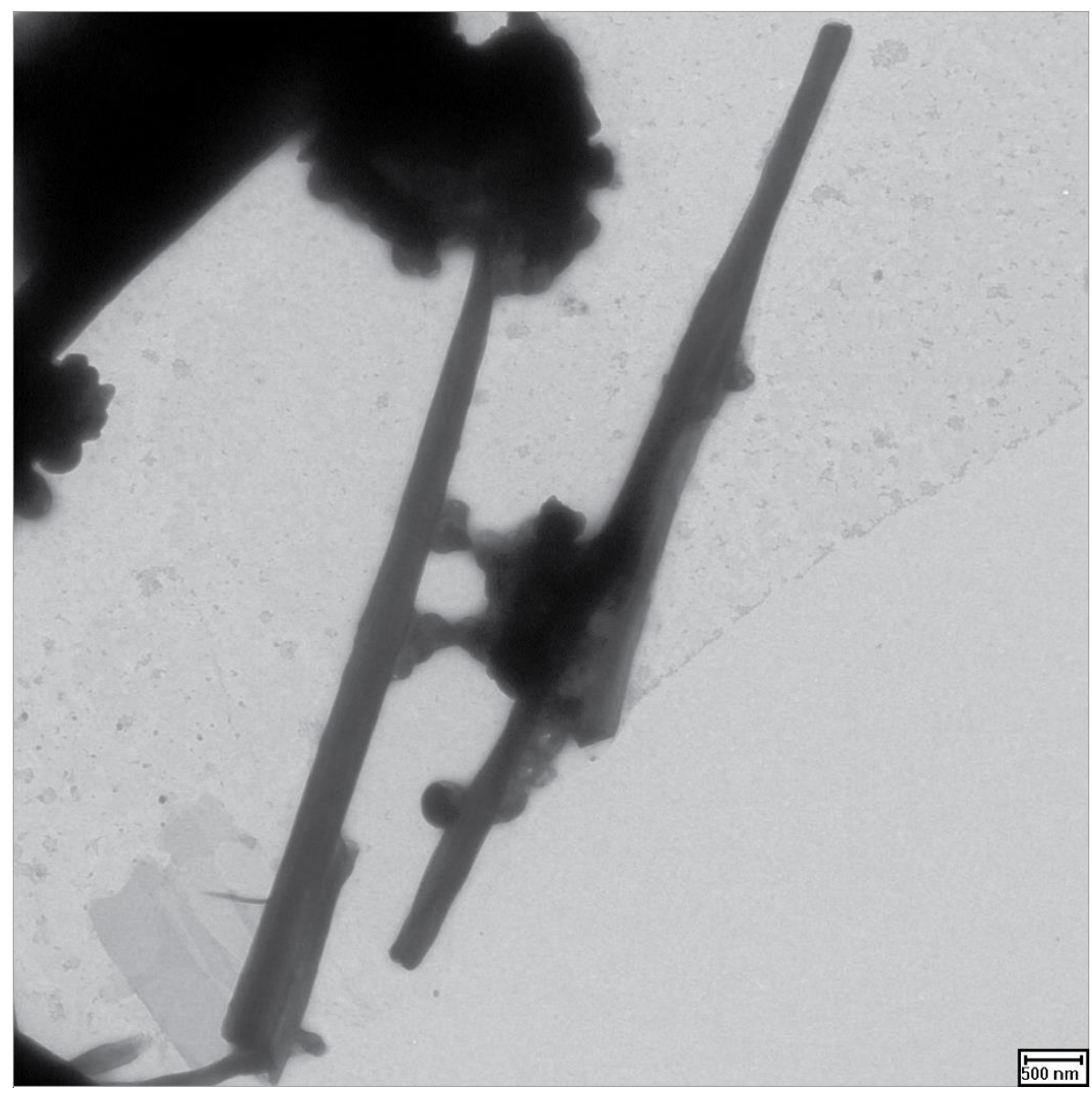

Figure S1: TEM micrograph showing long cylindrical cochleates. Sample was prepared in NanoAssemblr ${ }^{\mathrm{TM}}$ by mixing the $\mathrm{CaCl}_{2}$ buffer (in $10 \mathrm{mM}$ Tris) solution with DOPS liposomes $(1 \mathrm{mg} / \mathrm{ml}$ ) extruded through $100 \mathrm{~nm}$ polycarbonate membrane. Long cylindrical cochleate particles were prepared when DOPS was precipitated in complete absense of ethanol. Flow rate was $6 \mathrm{ml} / \mathrm{min}$ and mixing ratio for the two streams was 1:1. 


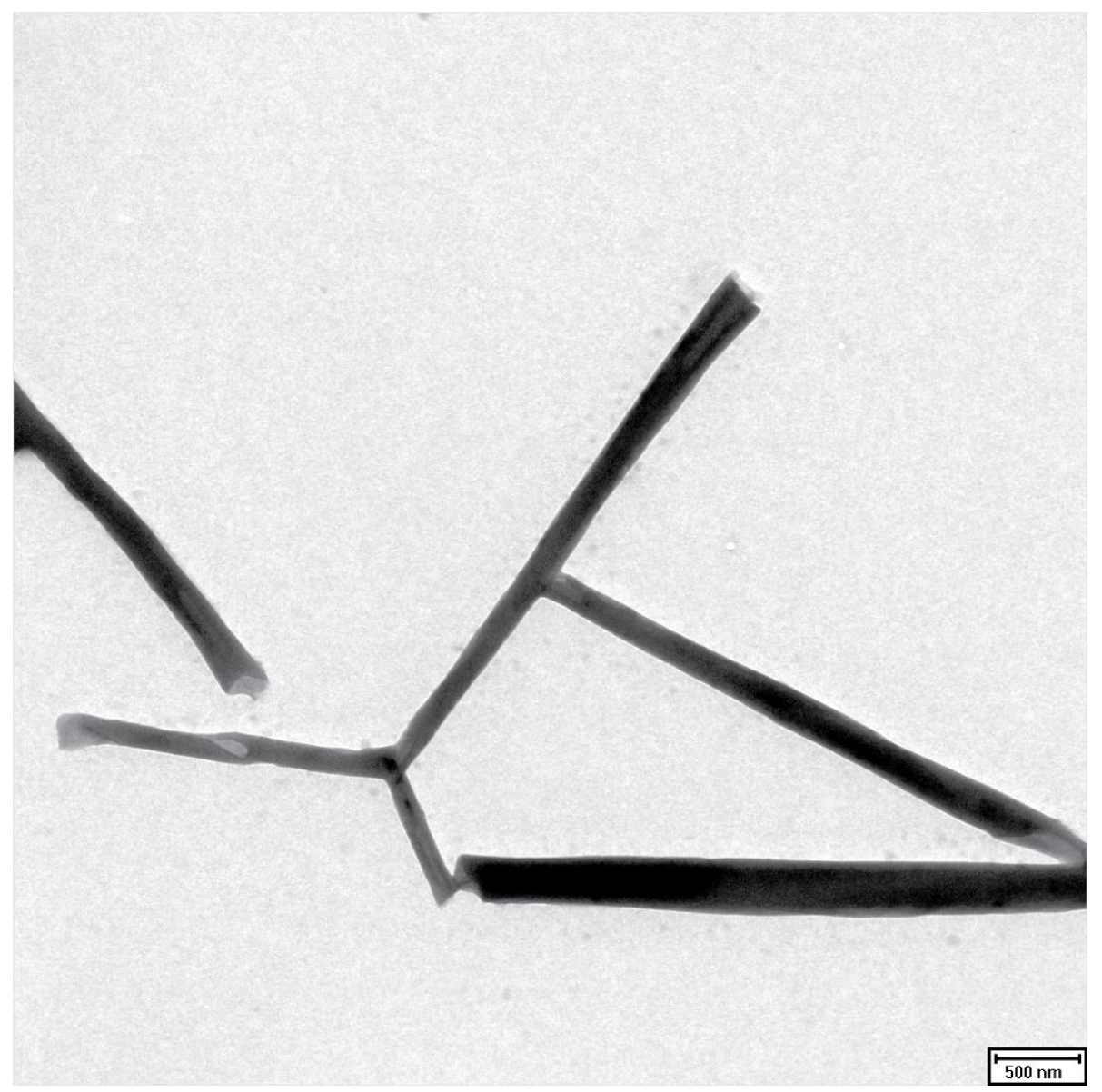

Figure S2: Long cylindrical cochleates observed when high aqueous component was used in the mixture. Ratio of $\mathrm{CaCl}_{2 \text { (aqueous) }}$ : DOPS (ethanolic) $(3: 1)$, mixing rate $12 \mathrm{ml} / \mathrm{min}$ and concentration of ethanolic DOPS was $1 \mathrm{mg} / \mathrm{ml}$. 


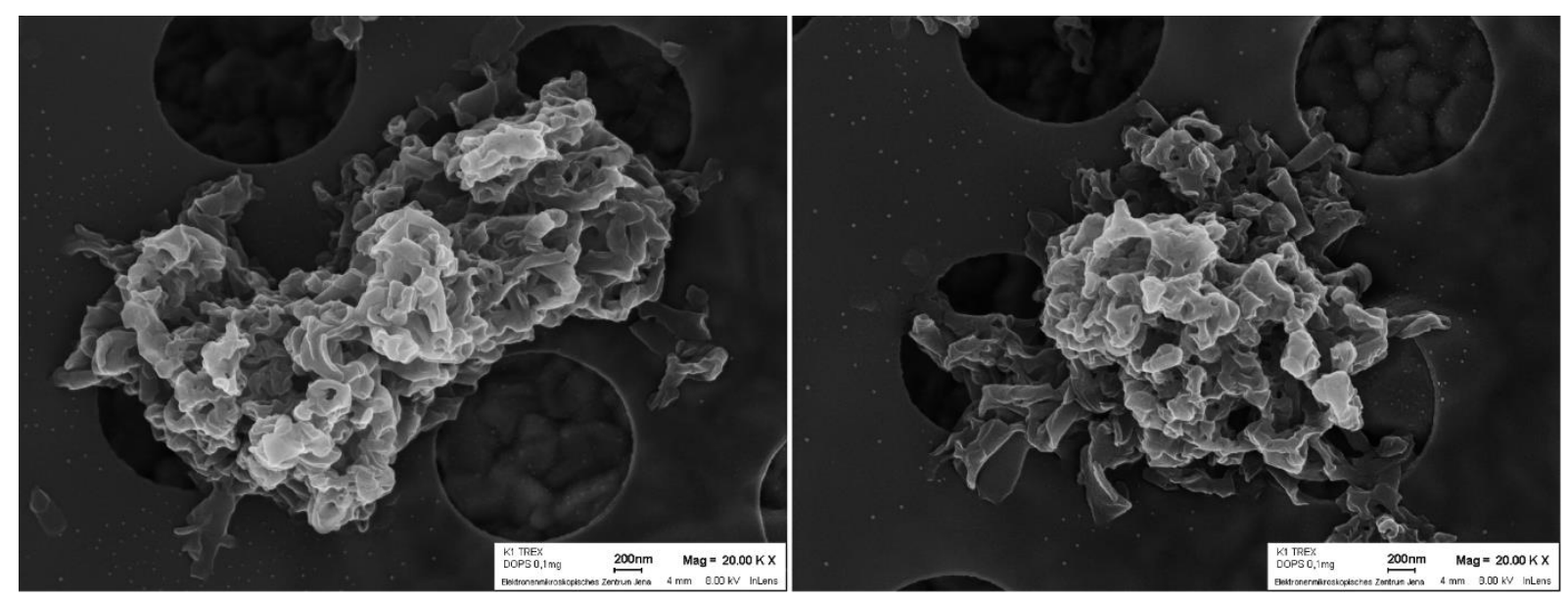

Figure S3: Cochleate composites prepared using the modified trapping method (using T-25 UltraTurrax ${ }^{\circledR}$ homogenizer) showed irregular non-spherical morphology. 


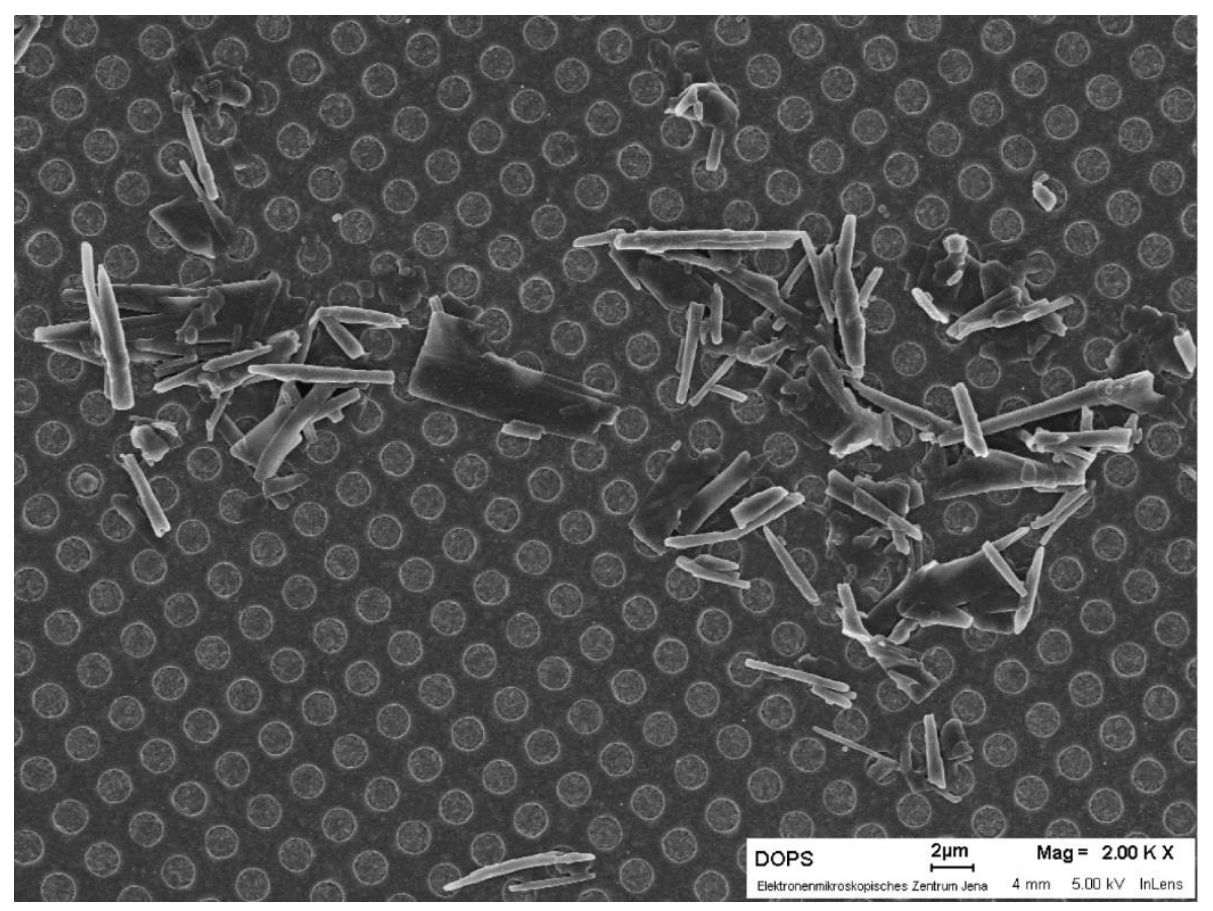

Figure S4: SEM micrograph showing an overview of the conventional DOPS cochleates prepared by trapping method. 